\title{
Hot Plasma Modes Across Reissner-Nördstrom de Sitter Horizon in a Veselago Medium
}

\author{
Ifra Noureen * \\ University of Management and Technology, Lahore, Pakistan. \\ H. Rizwana Kausar ${ }^{\dagger}$ \\ Director, Centre for Applicable Mathematics \& Statistics, \\ University of Central Punjab, Lahore, Pakistan.
}

\begin{abstract}
In this manuscript, wave attributes of hot plasma around ReissnerNördstrom-de Sitter (RN-dS) metric in a Veselago medium are investigated. General relativistic magnetohydrodynamical (GRMHD) equations in planar coordinates for the RN-dS horizon are reformulated by implementation of ADM $3+1$ technique. Further, perturbation scheme is used to arrive at linearly perturbed GRMHD equations whose component form is used to attain dispersion relations for rotating (non-magnetized and magnetized) plasma. Wave propagation in hot plasma is explained by three dimensional graphical representation of the wave number, refractive index, its change with respect to angular velocity, phase and group velocities. Finally, comparison of wave properties is presented, results reassert the presence of Veselago medium.
\end{abstract}

Keywords: GRMHD equations; Veselago medium; RN-dS spacetime. PACS: 95.30.Sf; 95.30.Qd; 04.30.Nk

*ifra.noureen@gmail.com

†rizwa_math@yahoo.com 


\section{Introduction}

Celestial objects such as Black hole $(\mathrm{BH})$ are abundant in our universe. In black hole gravitational pull is much stronger that nothing can escape from its so-called horizon. Relativists suggest that death of massive stars or collapse of a supergiant star give birth to black holes. In 1916, Schwarzschild found the simplest black hole as an exact solution of field equations [1].

Plasma is a distinct fourth state of matter having positive and negatively charged particles that potently interact with electromagnetic fields [2]. The theory of magnetohydrodynamics (MHD) is formulated for the study of plasma flow under the influence of magnetic field. Magnetohydrodynamics in connection with gravity impressions is recognized as a distinct and authentic theory called general relativistic magnetohydrodynamics (GRMHD) [3]. Exact solution of the field equations for a charged object constitute the Reissner-Nördstrom spacetime. The de-Sitter metric is a vacuum solution of the field equations with a positive cosmological constant leading to the far future expanding universe [4]-6].

Reissner-Nördstrom-de Sitter (RN-dS) metric corresponds to spacetime solutions characterized by charge, mass and cosmological constant that are assumed to be most pragmatic for astrophysical applications [7]. Regge and Wheeler [8], Zerilli [9] and Gleiser et al. [10] contributed to establish the stability of non-rotating black holes. ADM $3+1$ formalism[11] is the most reliable approach for spacetime decomposition in General relativity, it divides metric into layers of three-dimensional spacelike hypersurfaces and onedimensional time. Petterson [12] concluded that gravitational field is much stronger across the surface of non-rotating black hole.

Zhang [13] modified the laws of perfect GRMHD in general and also emphasized on stationary symmetric GRMHD solutions. He [14] also discuss the rotating black hole dynamics. Buzzi et al. [15] used ADM $3+1$ split to characterize two fluid plasma with the help of two local approximations in neighborhood of Schwarzschild black hole.

Astefanesei et al. [16] worked out Reissner-Nördstrom-de Sitter black holes in context of de-Sitter background to conformal field theory by using static and planar coordinates. Zhong and Gao [17] investigated particle collisions around cosmological horizon of a Reissner-Nordstrm de Sitter solution. Transverse wave propagation for two fluid plasma has also been acquired for Schwarzschild de-Sitter (SdS) magnetosphere [18]. Sharif and Sheikh [19][20] analyzed plasma modes of cold and isothermal plasmas in the usual 
medium for non-rotating and rotating black holes. Sharif and Rafique [21] presented dispersion modes of Schwarzschild horizon for the hot plasma in usual medium.

Metamaterials are artificially produced materials with unusual electromagnetic properties having enormous astrophysical applications. Russian physicist Victor Veselago introduced such a metamaterial named Veselago medium that have negative refractive index, permittivity and permeability simultaneously [22]. Various scientists [23]-[27] analyzed negative phase velocity and refractive index of such metamaterial. Ziolkowski and Heyman [28] developed analytic and numerical solution to establish wave properties of such medium. Ramakrishna [29] explained the role of Veselago medium in perfect lensing phenomenon. After experimental realization of veselago medium, plasma study in Veselago medium has gained enormous importance in recent years.

Sharif and Mukhtar [30] discussed dispersion relations for Schwarzschild horizon in unusual medium. Sharif and Noureen [31] determined dispersion modes of isothermal and hot plasma across SdS horizon for negative index medium. Recently, wave behavior around Reissner-Nördstrom spacetime is worked out [33].

We also use ADM $3+1$ split general line element

$$
d s^{2}=-\alpha^{2} d t^{2}+\eta_{m n}\left(d x^{m}+\beta^{m} d t\right)\left(d x^{n}+\beta^{n} d t\right),
$$

where lapse function (ratio of fiducial observer (FIDO), i.e, proper time to universal time, $\frac{d \tau}{d t}$ ) is expressed by $\alpha, \beta^{m}$ denotes shift vector in three dimensions and components of spacelike hypersurfaces are represented by $\eta_{m n}(m, n=1,2,3)$. In planar analogue of RN-dS spacetime $\alpha=\alpha(z), \quad \beta=$ $0, \quad \eta_{m n}=1(m=n)$ and so metric turn out to be

$$
d s^{2}=-\alpha^{2}(z) d t^{2}+d x^{2}+d y^{2}+d z^{2} .
$$

Wave analysis of hot plasma around RN-dS horizon in Veselago medium is examined in this paper. Since plasma in nature is highly ionized, so wave analysis of plasma around expanding charged spacetime is very captivating. The paper is arranged in the following manner: Fourier analyzed form of GRMHD equations for rotating black hole are given in section $\mathbf{2}$. Section 3 provides numerical solutions of linearly perturbed Fourier analyzed GRMHD equations for hot plasma. Comparison and summary is given in the last section. 


\section{Fourier Analyzed GRMHD Equations in a Veselago Medium}

Here, we use basic GRMHD equations (given in appendix) to get insight of Fourier analyzed GRMHD equations. It is assumed that plasma flow is two dimensional, i.e., in $x z$-plane. Velocity $\mathbf{V}$ and magnetic field $\mathbf{B}$ measured by FIDO are

$$
\mathbf{V}=v(z) \mathbf{e}_{x}+u(z) \mathbf{e}_{z}, \quad \mathbf{B}=B\left[\xi(z) \mathbf{e}_{x}+\mathbf{e}_{z}\right] .
$$

Here $B$ denotes constant. The relation between the quantities $\xi, u$ and $v$ is 19

$$
v=\frac{C}{\alpha}+\xi u
$$

where $C$ represents constant of integration. The Lorentz factor, $\gamma=\frac{1}{\sqrt{1-\mathbf{V}^{2}}}$ turn out to be $\gamma=\frac{1}{\sqrt{1-u^{2}-v^{2}}}$. In order to study the consequences of black hole gravity on plasma, we implement linear perturbation scheme to the flow variables (pressure $p$, mass density $\rho, \mathbf{V}$ and $\mathbf{B}$ ) as follows

$$
\begin{aligned}
& \rho=\rho^{0}+\epsilon \rho=\rho^{0}+\rho \widetilde{\rho}, \quad p=p^{0}+\epsilon p=p^{0}+p \widetilde{p} \\
& \mathbf{V}=\mathbf{V}^{0}+\epsilon \mathbf{V}=\mathbf{V}^{0}+\mathbf{v}, \mathbf{B}=\mathbf{B}^{0}+\epsilon \mathbf{B}=\mathbf{B}^{0}+B \mathbf{b}
\end{aligned}
$$

where $p, \rho^{0}, \mathbf{V}^{0}$ and $\mathbf{B}^{0}$ represent the unperturbed quantities. The linearly perturbed quantities are denoted by $\epsilon \rho, \epsilon p, \epsilon \mathbf{V}$ and $\epsilon \mathbf{B}$. Dimensionless quantities $\widetilde{\rho}, \widetilde{p}, v_{x}, v_{z}, b_{x}$ and $b_{z}$ are inserted which corresponds to perturbed quantities

$$
\begin{aligned}
& \tilde{p}=\tilde{p}(t, z), \quad \tilde{\rho}=\tilde{\rho}(t, z), \quad \mathbf{v}=\epsilon \mathbf{V}=v_{x}(t, z) \mathbf{e}_{x}+v_{z}(t, z) \mathbf{e}_{z}, \\
& \mathbf{b}=\frac{\epsilon \mathbf{B}}{B}=b_{x}(t, z) \mathbf{e}_{x}+b_{z}(t, z) \mathbf{e}_{z} .
\end{aligned}
$$

Application of linear perturbation in perfect GRMHD equations (A1)-(A5) and insertion of Eq.(2.6) leads to component form of linearly perturbed GRMHD equations [31, provided in the Appendix. Further, to take Fourier analysis of perturbed GRMHD equations, metric dependence is considered in the following way

$$
\begin{aligned}
\tilde{\rho}(t, z) & =a_{1} e^{-\iota \omega t+\iota k z}, & & \tilde{p}(t, z)=a_{2} e^{-\iota \omega t+\iota k z}, \\
v_{z}(t, z) & =a_{3} e^{-\iota \omega t+\iota k z}, & & v_{x}(t, z)=a_{4} e^{-\iota \omega t+\iota k z}, \\
b_{z}(t, z) & =a_{5} e^{-\iota \omega t+\iota k z}, & & b_{x}(t, z)=a_{6} e^{-\iota \omega t+\iota k z},
\end{aligned}
$$


where $k$ is $z$-component of the wave vector (wave number), angular frequency is expressed by $\omega$. To find refractive index and other plasma properties, we use above wave number. Here dispersion corresponding to the effects of frequency dependence during wave propagation. Insertion of Eq.(2.7) and component form of linearly perturbed GRMHD equations leads to the Fourier analyzed form,

$$
\begin{aligned}
& a_{4}\left(\alpha^{\prime}+\iota k \alpha\right)-a_{3}\left\{(\alpha \xi)^{\prime}+\iota k \alpha \xi\right\}-a_{5}(\alpha v)^{\prime}-a_{6}\left\{(\alpha u)^{\prime}+\iota \omega\right. \\
& +\iota k u \alpha\}=0 \text {, } \\
& a_{5}\left(\frac{-\iota \omega}{\alpha}\right)=0 \\
& a_{5} \iota k=0 \text {, } \\
& a_{1}\left(\frac{-\iota \omega}{\alpha} \rho\right)+a_{2}\left(\frac{-\iota \omega}{\alpha} p\right)+a_{3}(\rho+p)\left[\frac{-\iota \omega}{\alpha} \gamma^{2} u+\left(1+\gamma^{2} u^{2}\right) \iota k\right. \\
& \left.-\left(1-2 \gamma^{2} u^{2}\right)\left(1+\gamma^{2} u^{2}\right) \frac{u^{\prime}}{u}+2 \gamma^{4} u^{2} v v^{\prime}\right]+a_{4}(\rho+p) \gamma^{2}\left[\left(\frac{-\iota \omega}{\alpha}\right.\right. \\
& \left.+\iota k u) v+u\left(1+2 \gamma^{2} v^{2}\right) v^{\prime}+2 \gamma^{2} u^{2} v u^{\prime}\right]=0, \\
& a_{1}\left[\rho \gamma^{2} u\left\{\left(1+\gamma^{2} v^{2}\right) v^{\prime}+\gamma^{2} v u u^{\prime}\right\}+\gamma^{2} v u\left(\rho^{\prime}+\iota k \rho\right)\right]+a_{2}\left[p \gamma^{2} u\right. \\
& \left.\times\left\{\left(1+\gamma^{2} v^{2}\right) v^{\prime}+\gamma^{2} v u u^{\prime}\right\}+\gamma^{2} v u\left(p^{\prime}+\iota k p\right)\right]+a_{3}\left[(\rho+p) \gamma^{2}\{(1\right. \\
& \left.+2 \gamma^{2} u^{2}\right)\left(1+2 \gamma^{2} v^{2}\right) v^{\prime}+\left(\frac{-\iota \omega}{\alpha}+\iota k u\right) \gamma^{2} v u-\gamma^{2} v^{2} v^{\prime}+2 \gamma^{2}(1+ \\
& \left.\left.2 \gamma^{2} u^{2}\right) u v u^{\prime}\right\}+\gamma^{2} v\left(1+2 \gamma^{2} u^{2}\right)\left(\rho^{\prime}+p^{\prime}\right)-\frac{B^{2} u}{4 \pi \alpha}(\xi \alpha)^{\prime}+\frac{\xi B^{2}}{4 \pi}\left(\frac{\iota \omega}{\alpha}\right. \\
& -\iota k u)]+a_{4}\left[(\rho+p) \gamma^{4} u\left\{\left(1+4 \gamma^{2} v^{2}\right) u u^{\prime}+4 v v^{\prime}\left(1+\gamma^{2} v^{2}\right)\right\}+(\rho+\right. \\
& \text { p) } \gamma^{2}\left(1+\gamma^{2} v^{2}\right)\left(\frac{-\iota \omega}{\alpha}+\iota k u\right)+\gamma^{2} u\left(1+2 \gamma^{2} v^{2}\right)\left(\rho^{\prime}+p^{\prime}\right)+\frac{B^{2}}{4 \pi}\left(\frac{u \alpha^{\prime}}{\alpha}-\right. \\
& \left.\left.\frac{\iota \omega}{\alpha}+\iota k u\right)\right]-a_{6} \frac{B^{2}}{4 \pi \alpha}\left[\alpha u u^{\prime}+\alpha^{\prime}\left(1+u^{2}\right)+\left(1+u^{2}\right) \iota k \alpha\right]=0, \\
& a_{1}\left[\rho \gamma^{2}\left\{a_{z}+\left(1+\gamma^{2} u^{2}\right) u u^{\prime}+\gamma^{2} u^{2} v v^{\prime}\right\}+\gamma^{2} u^{2}\left(\rho^{\prime}+\iota k \rho\right)\right]+a_{2}\left[p \gamma ^ { 2 } \left\{a_{z}\right.\right. \\
& \left.\left.+\left(1+\gamma^{2} u^{2}\right) u u^{\prime}+\gamma^{2} u^{2} v v^{\prime}\right\}+\left(1+\gamma^{2} u^{2}\right)\left(p^{\prime}+\iota k p\right)\right]+a_{3}\left[(\rho+p) \gamma^{2}\{(1\right. \\
& \left.+\gamma^{2} u^{2}\right)\left(\frac{-\iota \omega}{\alpha}+\iota k u\right)+u^{\prime}\left(1+\gamma^{2} u^{2}\right)\left(1+4 \gamma^{2} u^{2}\right)+2 u \gamma^{2}\left(a_{z}+\left(1+2 \gamma^{2} u^{2}\right)\right. \\
& \left.\left.\left.\times v v^{\prime}\right)\right\}+2 \gamma^{2} u\left(1+\gamma^{2} u^{2}\right)\left(\rho^{\prime}+p^{\prime}\right)+\frac{\xi B^{2} u}{4 \pi \alpha}(\xi \alpha)^{\prime}-\frac{\xi^{2} B^{2}}{4 \pi}\left(\frac{\iota \omega}{\alpha}-\iota k u\right)\right] \\
& +a_{4}\left[( \rho + p ) \gamma ^ { 4 } \left\{\left(\frac{-\iota \omega}{\alpha}+\iota k u\right) u v+u^{2} v^{\prime}\left(1+4 \gamma^{2} v^{2}\right)+2 v\left(a_{z}+\right.\right.\right.
\end{aligned}
$$




$$
\begin{aligned}
& \left.\left.\left.\left(1+2 \gamma^{2} u^{2}\right) u u^{\prime}\right)\right\}+2 \gamma^{4} u^{2} v\left(\rho^{\prime}+p^{\prime}\right)+\frac{\xi B^{2}}{4 \pi}\left(\frac{\iota \omega}{\alpha}-\iota k u\right)-\frac{\xi B^{2} u \alpha^{\prime}}{4 \pi \alpha}\right]+ \\
& a_{6}\left[\frac{B^{2}}{4 \pi \alpha}\left\{-(\xi \alpha)^{\prime}+\alpha^{\prime} \xi-u \xi\left(u \alpha^{\prime}+u^{\prime} \alpha\right)\right\}+\frac{\xi B^{2}}{4 \pi}\left(1+u^{2}\right) \iota k\right]=0, \\
& a_{1}\left\{\left(\frac{-\iota \omega}{\alpha} \gamma^{2}+\iota k u \gamma^{2}+2 u \gamma^{2} a_{z}+\gamma^{2} u^{\prime}\right) \rho+u \rho^{\prime} \gamma^{2}\right\}+a_{2}\left\{\left(\frac{\iota \omega}{\alpha}\left(1-\gamma^{2}\right)\right.\right. \\
& \left.\left.+\iota k u \gamma^{2}+2 \gamma^{2} u a_{z}+\gamma^{2} u^{\prime}\right) p+u \gamma^{2} p^{\prime}\right\}+a_{3} \gamma^{2}\left\{\left(\rho^{\prime}+p^{\prime}\right)+2\left(2 \gamma^{4} u u^{\prime}+\right.\right. \\
& \left.\left.a_{z}+2 \gamma^{2} u^{2} a_{z}\right)(\rho+p)+\left(1+2 \gamma^{2} u^{2}\right)(\rho+p) \iota k+\frac{\xi B^{2}}{4 \pi \alpha}(\xi u-v) \iota \omega+\alpha \xi^{\prime}\right\} \\
& +a_{4}\left[2(\rho+p) \gamma^{2}\left\{\left(u v^{\prime}+2 u v a_{z}+u^{\prime} v\right)+u v \iota k\right\}+\frac{B^{2}}{4 \pi \alpha}(v-u \xi) \iota \omega-\alpha \xi^{\prime}\right]+ \\
& a_{6}\left[\frac{-B^{2}}{4 \pi \alpha}\left\{\left(v^{2}+u^{2}\right) \xi+\xi v(\xi v+u) \iota \omega\right\}-\alpha \xi^{\prime} u+\iota k \alpha(v-u \xi)\right]=0 .
\end{aligned}
$$

\section{Hot plasma dispersion modes}

The equation of state is used to obtain the numerical solution of Fourier analyzed form for rotating environment. For hot plasma, it is given by [13]

$$
\mu=\frac{\rho+p}{\rho_{0}}
$$

The specific enthalpy $\mu$ in Eq.(3.15) is non-constant, so affects the perturbed GRMHD equations (2.8)-(2.14) for hot plasma around the RN-dS black horizon. In the following subsections, we discuss the hot plasma case in the magnetized and non-magnetized backgrounds.

\subsection{Non-magnetized Background}

In non-magnetized environment, $B=0=\xi$ leads to $a_{5}=0=a_{6}$, in the Fourier analyzed GRMHD equations (2.11)-(2.14) [31]. In order to solve Fourier analyzed equations numerically, we assume $\mu=\sqrt{\frac{1+\alpha^{2}}{2}}$ and $\rho=p=$ $\frac{\mu}{2}, \alpha=\frac{z}{2 r}, r=r_{+}+r_{h}$, with $r_{+}=\frac{z}{2\left(M+\sqrt{M^{2}+Q^{2}}\right)}, \frac{Q^{2}}{M^{2}}=0.7, u=v=-\frac{1}{\sqrt{z^{2}+2}}$, and $\gamma=\frac{1}{\sqrt{1-u^{2}-v^{2}}}=\frac{\sqrt{z^{2}+2}}{z}$, where $r_{h} \approx 2 M\left(1+\frac{4 M^{2}}{l^{2}}+\ldots\right) \simeq \zeta 2.948 k m$, $1 \leqslant \zeta \leqslant 1.5$ and $M \sim 1 M_{\odot}[18$.

The region under consideration is $4 \leq z \leq 10$ with event horizon at $z=0$. The region $0 \leq z \leq 4$ is ignored because wave propagation can not be viewed 
graphically and result are not interesting in this region. A dispersion relation is formed from determinant of coefficients of the corresponding Fourier analyzed equations for non-magnetized plasma. Real part of dispersion relation evolve quartic equation in $k$, given as

$$
H_{1}(z) k^{4}+H_{2}(z, \omega) k^{3}+H_{3}(z, \omega) k^{2}+H_{4}(z, \omega) k+H_{5}(z, \omega)=0 .
$$

Above equation has two real and two imaginary roots. Imaginary part of dispersion relation is cubic in $k$ as follows

$$
D_{1}(z) k^{3}+D_{2}(z, \omega) k^{2}+D_{3}(z, \omega) k+D_{4}(z, \omega)=0 .
$$

One real and two complex roots are originated from imaginary part.

Wave number $k$ can be used to obtain refractive index whose change $\frac{d n}{d \omega}$ decides whether the dispersion is anomalous or normal, equivalently the region of greater phase velocity than the group velocity corresponds to normal dispersion, otherwise anomalous [32. Wave phenomenon around event horizon can be beneficial only for those values of $k$ for which waves propagate in opposite direction of the horizon and dispersion is normal. However, no information can be extracted about magnetosphere for waves that disperse anomalously.

It is to be mentioned here that dispersion relations are solved by using software Mathematica. There are few roots for which change in refractive index does not show three dimensional plot but its values are shown on axis. Some complicated expressions that mathematica could not plot in the form of graph causes this.

Figures 1-3 provide three dimensional view of wave properties of nonmagnetized hot plasma around RN-dS horizon, that are further explained in table I.

Table I. Dispersion and direction of waves

\begin{tabular}{|c|c|}
\hline In direction of horizon & Fig. 1,3 \\
\hline Moving away from horizon & Fig. 2 \\
\hline Normal dispersion & Fig. 3 \\
\hline Anomalous dispersion & Fig. 1,2 \\
\hline & Fig. $1,4.3 \leq z \leq 7.9,0 \leq \omega \leq 9$ \\
Increasing $n$ with & Fig. $2,4 \leq z \leq 9.2,0 \leq \omega \leq 10$ \\
increasing $z$ & Fig. $3,7.5 \leq z \leq 9,1 \leq \omega \leq 8$ \\
\hline
\end{tabular}



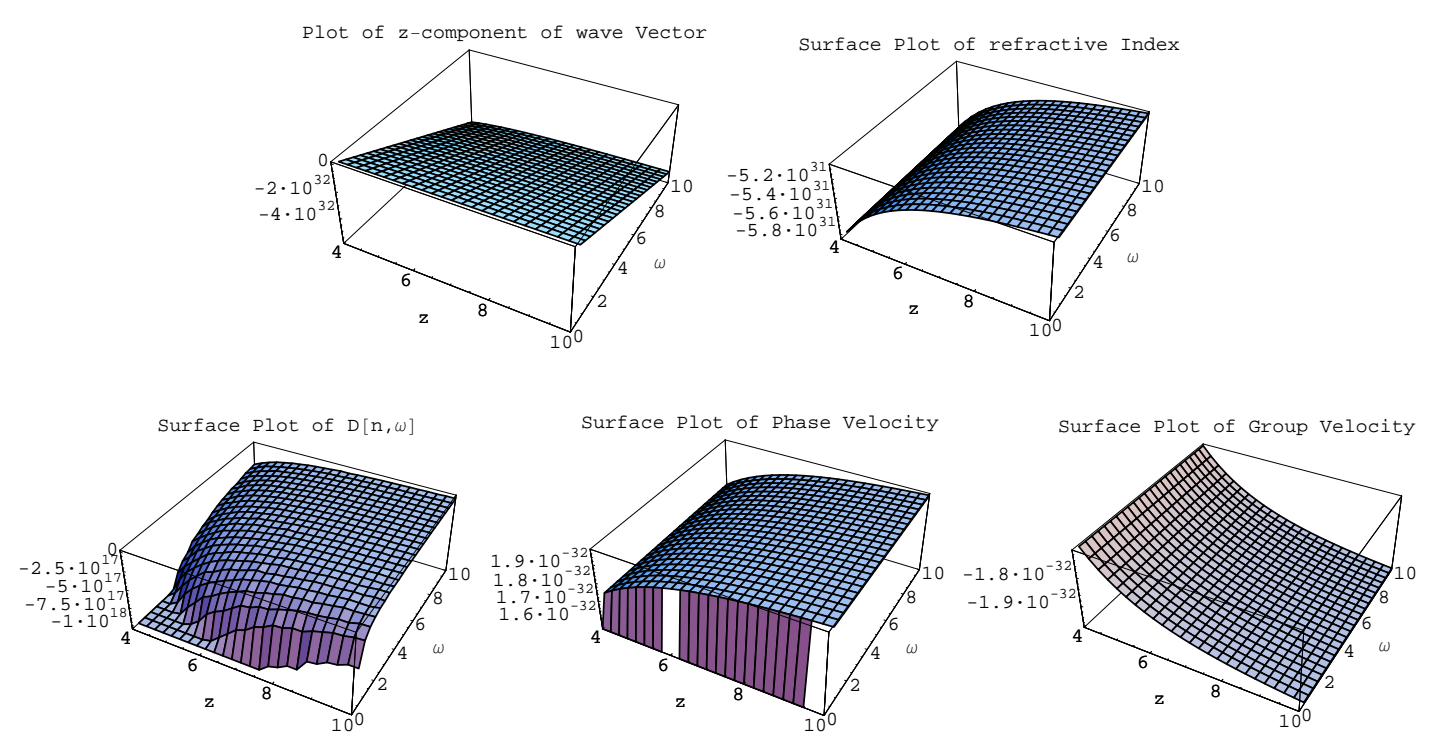

Figure 1: Dispersion is normal and anomalous in the region
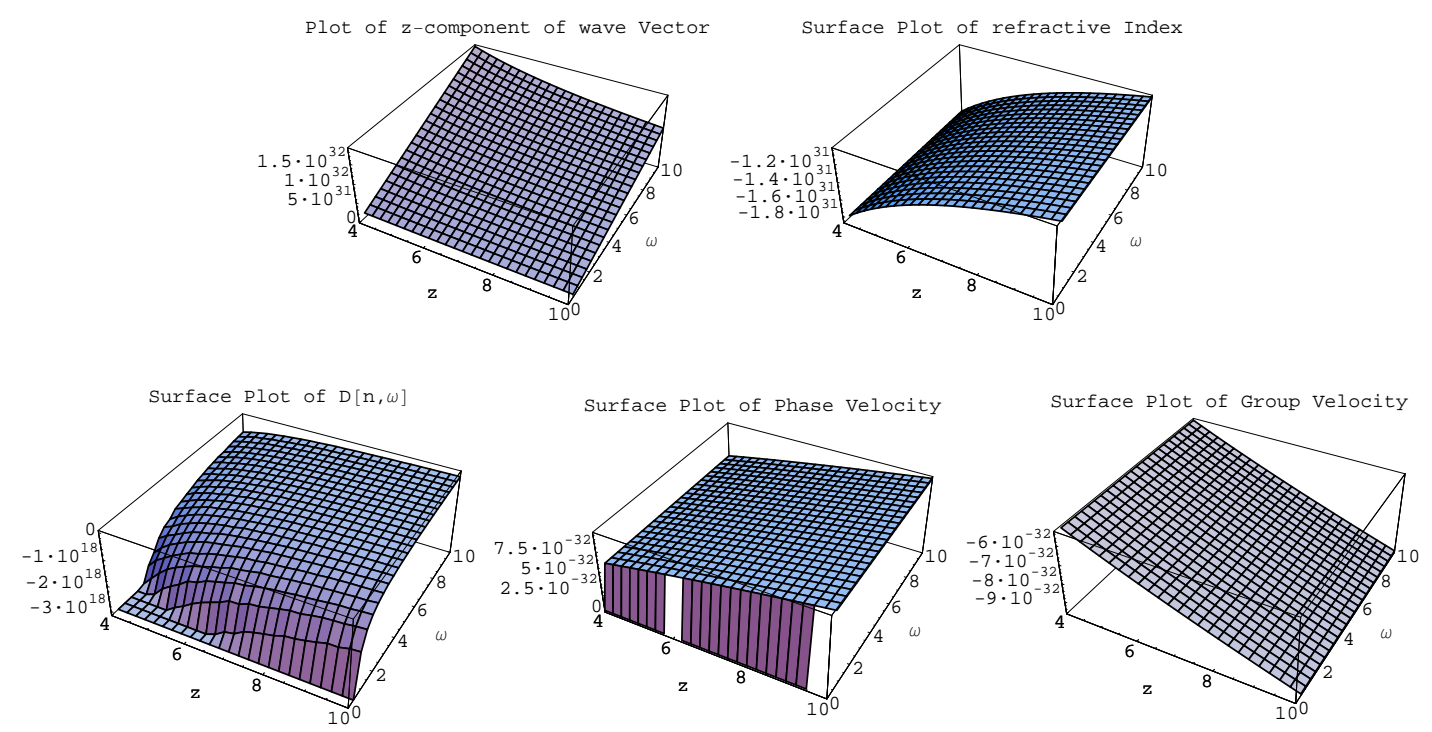

Figure 2: Normal as wells as anomalous dispersion occur at random points in the region. 

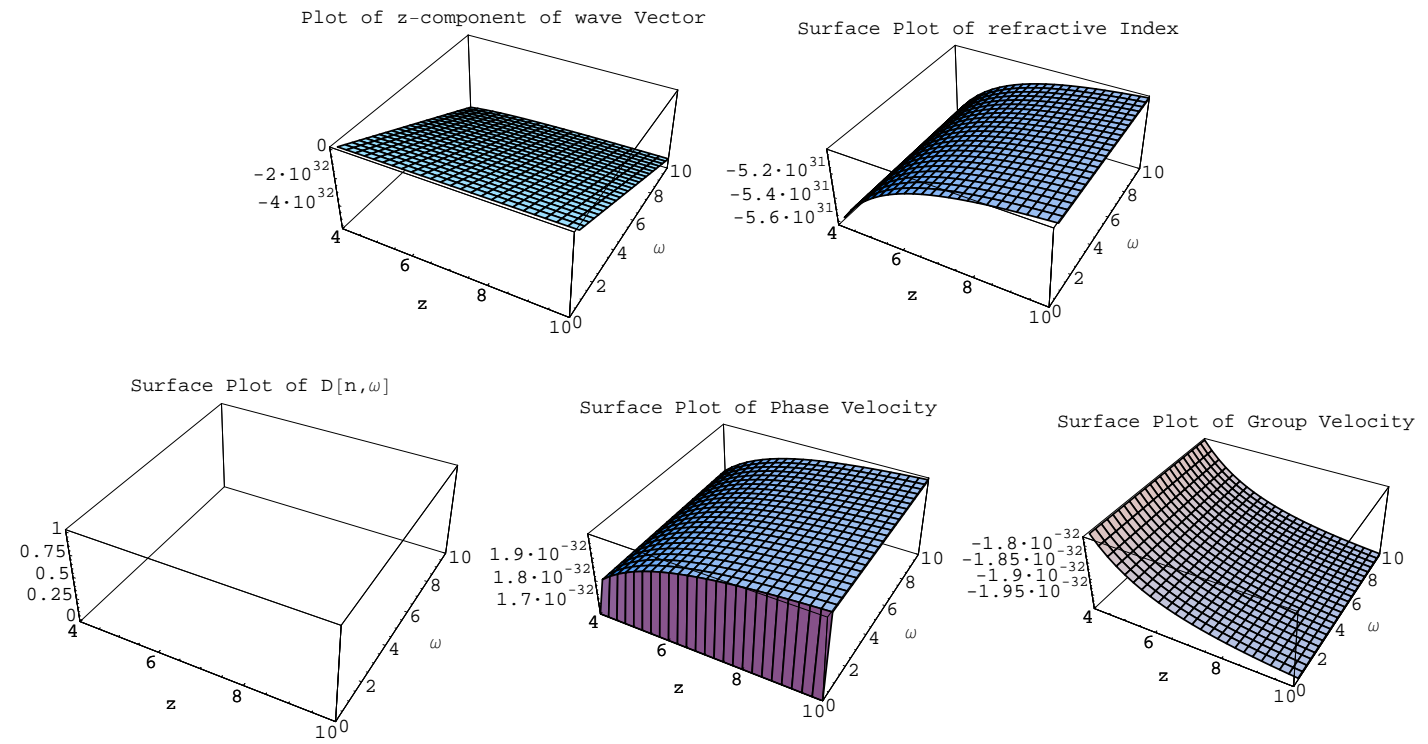

Figure 3: Normal and anomalous dispersion of waves is observed.

\subsection{Plasma flow in Magnetized Background}

For rotating magnetized plasma flow, the perturbed Fourier analyzed GRMHD equations are given by Eqs.(2.8)-(2.14). In this case, flow is two dimensional hence $\mathbf{V}$ and $\mathbf{B}$ lie in $x z$-plane. For numerical solutions velocity, lapse function and specific enthalpy are the same as for non-magnetized background. Here, magnetic field is assumed to be $\frac{B^{2}}{4 \pi}=2$ with $u=v$. By putting $C=1$ in Eq.(2.4), we have $\xi=1+\frac{\sqrt{2+z^{2}}}{z}$. Region for wave analysis is considered to be $4 \leq z \leq 10,0 \leq \omega \leq 10$ and in this case Eqs.(2.9)-(2.10) yields $a_{5}=0$. The determinant of Fourier analyzed form generates dispersion relation whose real part is as follows

$$
D_{1}(z) k^{4}+D_{2}(z, \omega) k^{3}+D_{3}(z, \omega) k^{2}+D_{4}(z, \omega) k+D_{5}(z, \omega)=0 .
$$

Four real roots originates from the real part of elaborated in Figures 4-7. Imaginary part is given by

$$
\begin{aligned}
& E_{1}(z) k^{5}+E_{2}(z, \omega) k^{4}+E_{3}(z, \omega) k^{3}+E_{4}(z, \omega) k^{2}+E_{5}(z, \omega) k \\
& +E_{6}(z, \omega)=0 .
\end{aligned}
$$

yield five roots, three real and two complex described by Figures 8-10. Plasma modes for magnetized background are tabulated in Table II and 

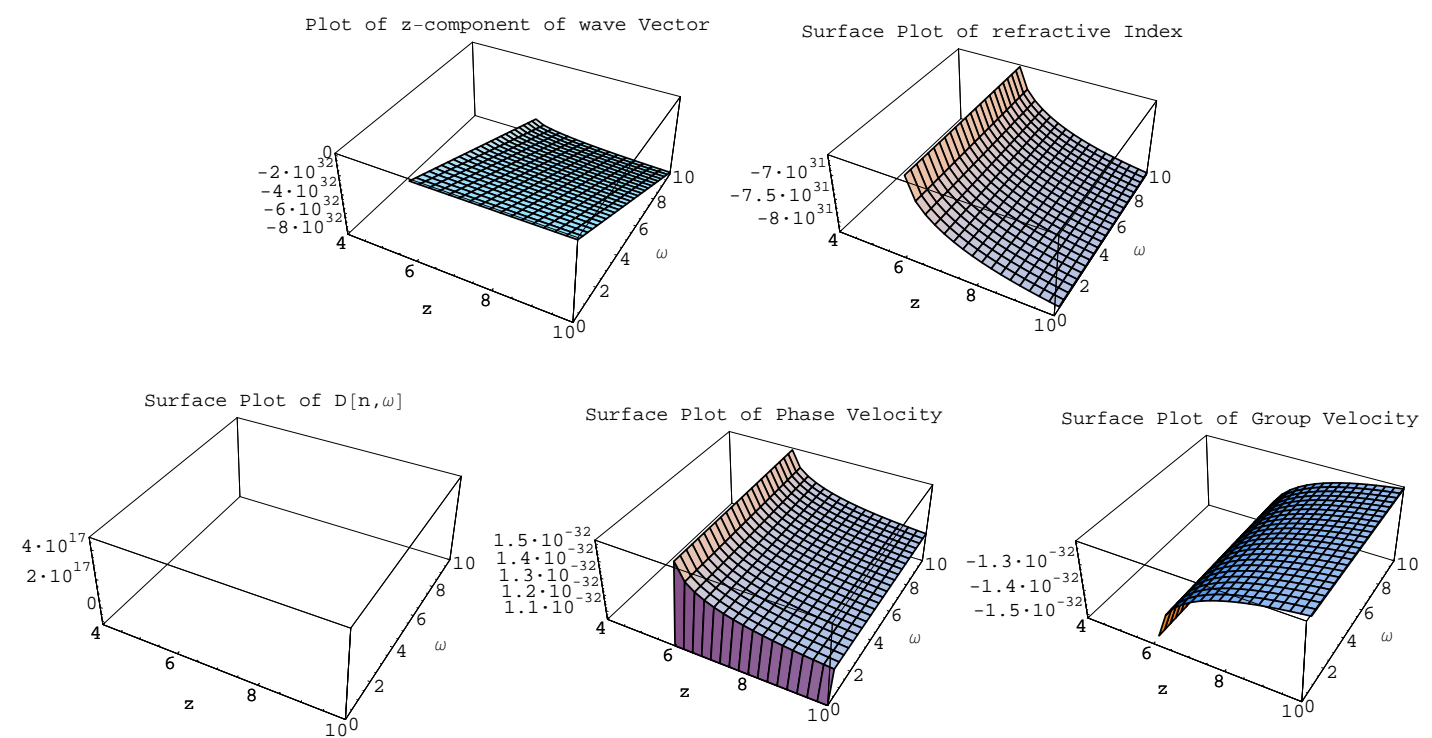

Figure 4: Random points of normal and anomalous dispersion are found in the region.

III.

Table II. Refractive index and direction of waves

\begin{tabular}{|c|c|}
\hline In direction of horizon & Fig. 4,5,6,8,9 \\
\hline Moving away from horizon & Fig. 7,10 \\
\hline Normal dispersion & Fig. 4 \\
\hline Anomalous dispersion & Fig. 5 \\
\hline & Fig. 5, $6.1 \leq z \leq 9.9,1 \leq \omega \leq 8$ \\
& Fig. $6,4.6 \leq z \leq 8.6,1 \leq \omega \leq 9.6$ \\
Increasing $n$ with & Fig. $7,4 \leq z \leq 8.2,0 \leq \omega \leq 9$ \\
increasing $z$ & Fig. $8,4.3 \leq z \leq 9.4,1 \leq \omega \leq 9$ \\
& Fig. $10,4 \leq z \leq 6.8,1 \leq \omega \leq 9$ \\
\hline Decreasing $n$ with & Fig. $4,5.7 \leq z \leq 10,0 \leq \omega \leq 4$ \\
increasing $z$ & Fig. $9,4.3 \leq z \leq 4.9,1.3 \leq \omega \leq 9.1$ \\
\hline
\end{tabular}

Table V. Normal and anomalous dispersion regions 

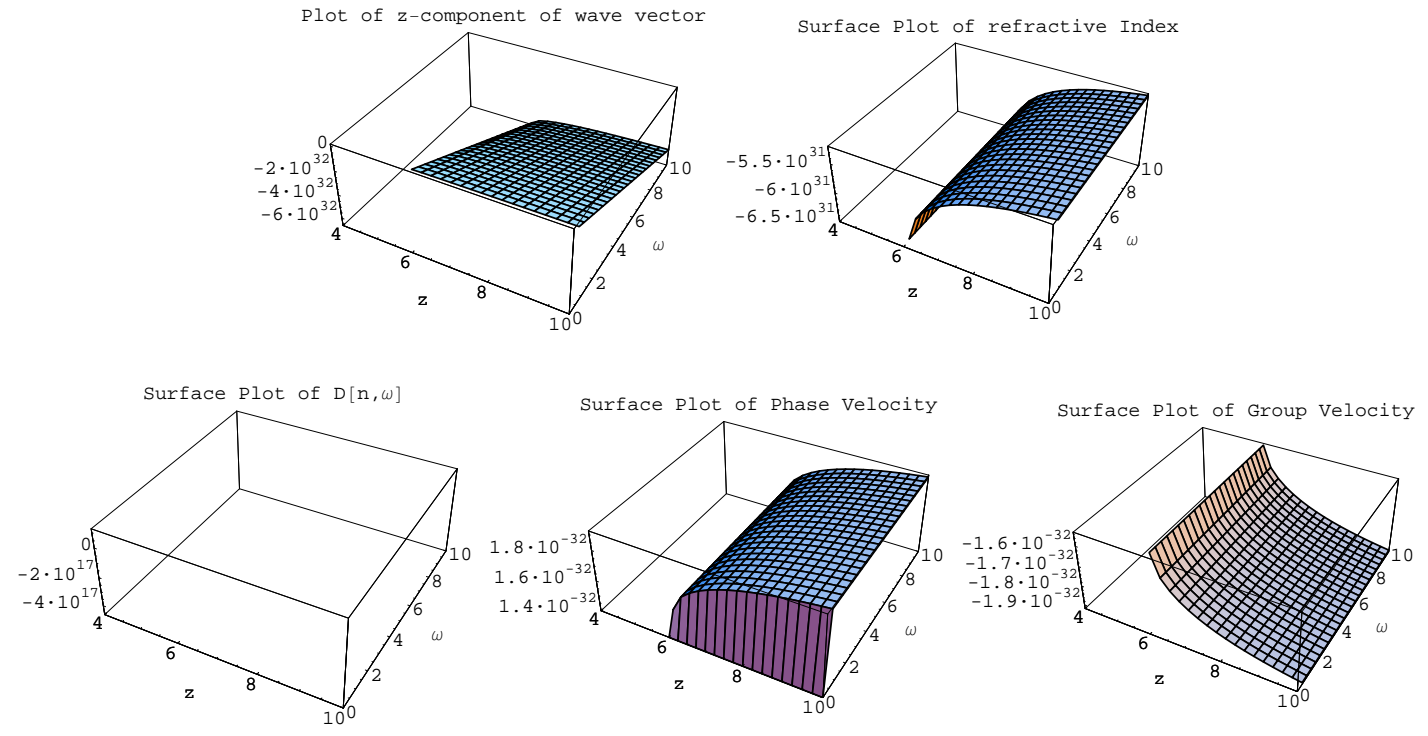

Figure 5: Whole region admits normal dispersion.

\begin{tabular}{|c|c|c|}
\hline Fig. & Normal dispersion & Anomalous dispersion \\
\hline $\mathbf{7}$ & $4 \leq z \leq 5.1,4 \leq \omega \leq 6$ & $4.9 \leq z \leq 5.3,0 \leq \omega \leq 1.7$ \\
\hline $\mathbf{8}$ & $8.1 \leq z \leq 8.9,1.5 \leq \omega \leq 3.1$ & $4.3 \leq z \leq 5.1,7 \leq \omega \leq 8.3$ \\
\hline $\mathbf{9}$ & $5.3 \leq z \leq 5.4,1.8 \leq \omega \leq 1.9$ & $5.1 \leq z \leq 5.7,7 \leq \omega \leq 8$ \\
\hline & $4.1 \leq z \leq 4.4,1.5 \leq \omega \leq 3$ & $7 \leq z \leq 7.9, .5 \leq \omega \leq 1.1$ \\
$\mathbf{1 0}$ & $7 \leq z \leq 7.7,4.2 \leq \omega \leq 4.9$ & \\
\hline & $4 \leq z \leq 4.4,7 \leq \omega \leq 7.7$ & $8 \leq z \leq 8.6,9.2 \leq \omega \leq 9.4$ \\
$\mathbf{1 2}$ & & $4 \leq z \leq 4.3,0 \leq \omega \leq .3$ \\
\hline
\end{tabular}

\section{Summary}

Plasma modes of hot plasma around RN-dS horizon in the presence of Veselago medium are investigated in this paper. We rewrite perfect GRMHD equations in Veselago medium by implementing ADM formalism. Plasma flow distracts due to gravitational effects of black hole. Linear perturbation is applied on flow variables to investigate the gravity effects of black hole. Moreover, it is assumed that plasma flow is two dimensional, i.e., xz-plane. Dimensionless quantities corresponding to flow variables are introduced to build component form of linearly perturbed GRMHD equations. 

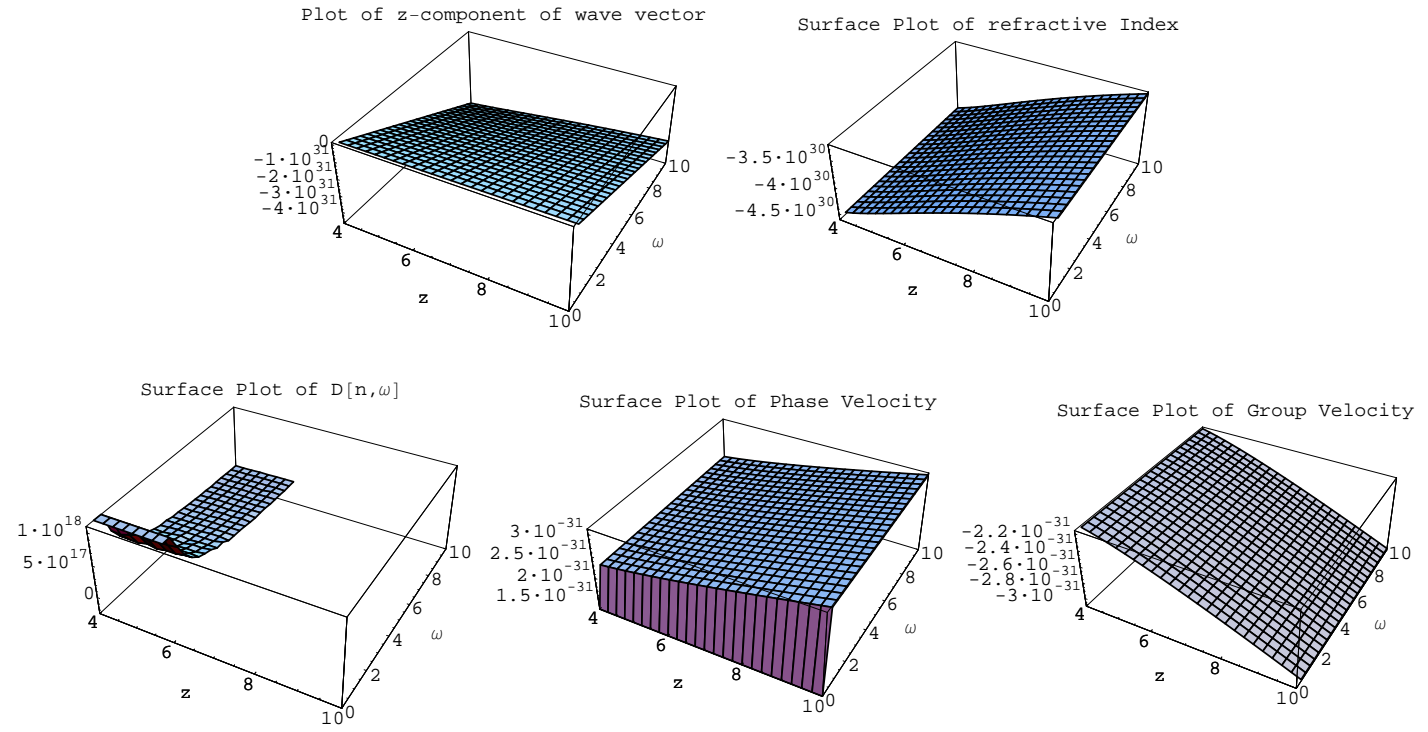

Figure 6: Normal and anomalous dispersion of waves is observed.
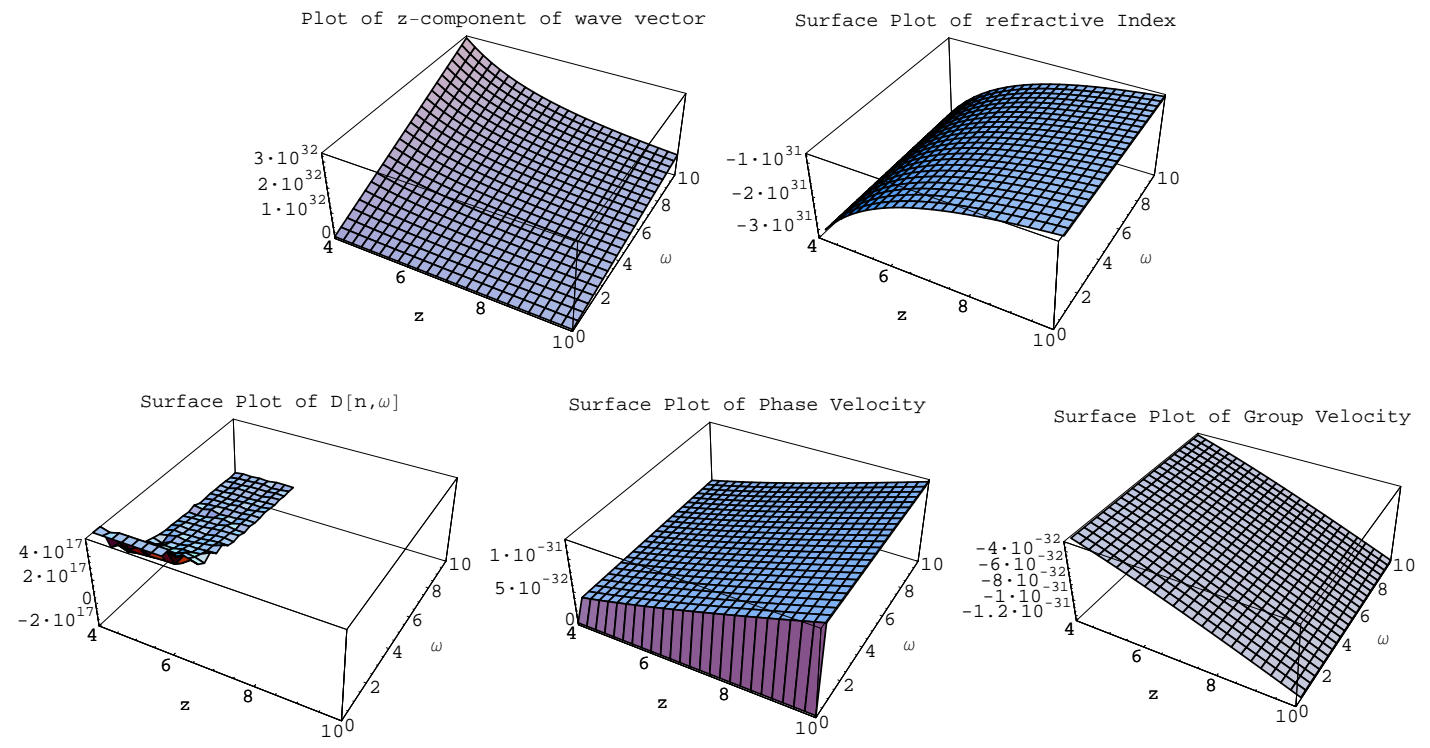

Figure 7: Waves disperse normally in the whole region. 

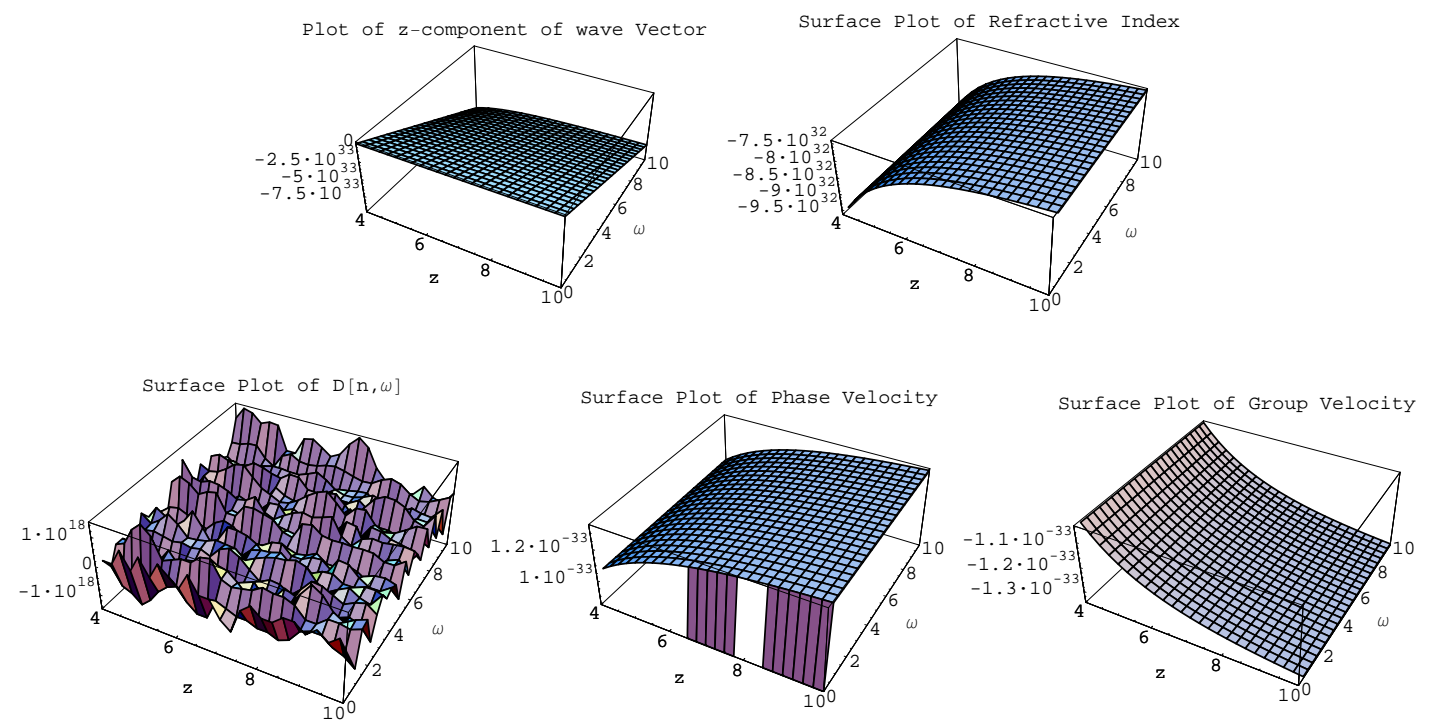

Figure 8: Waves exhibit normal as well as normal dispersion at random points.
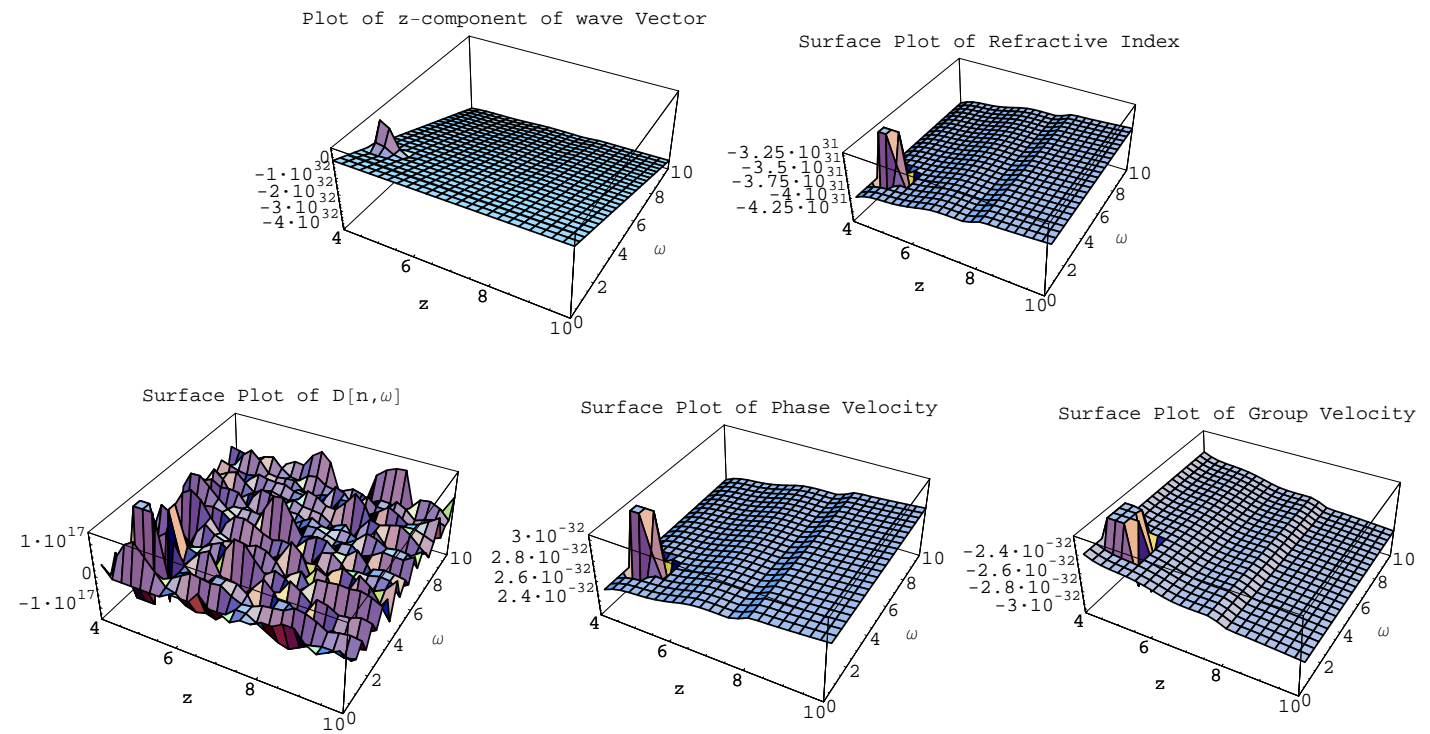

Figure 9: Waves disperse normally in the whole region. 

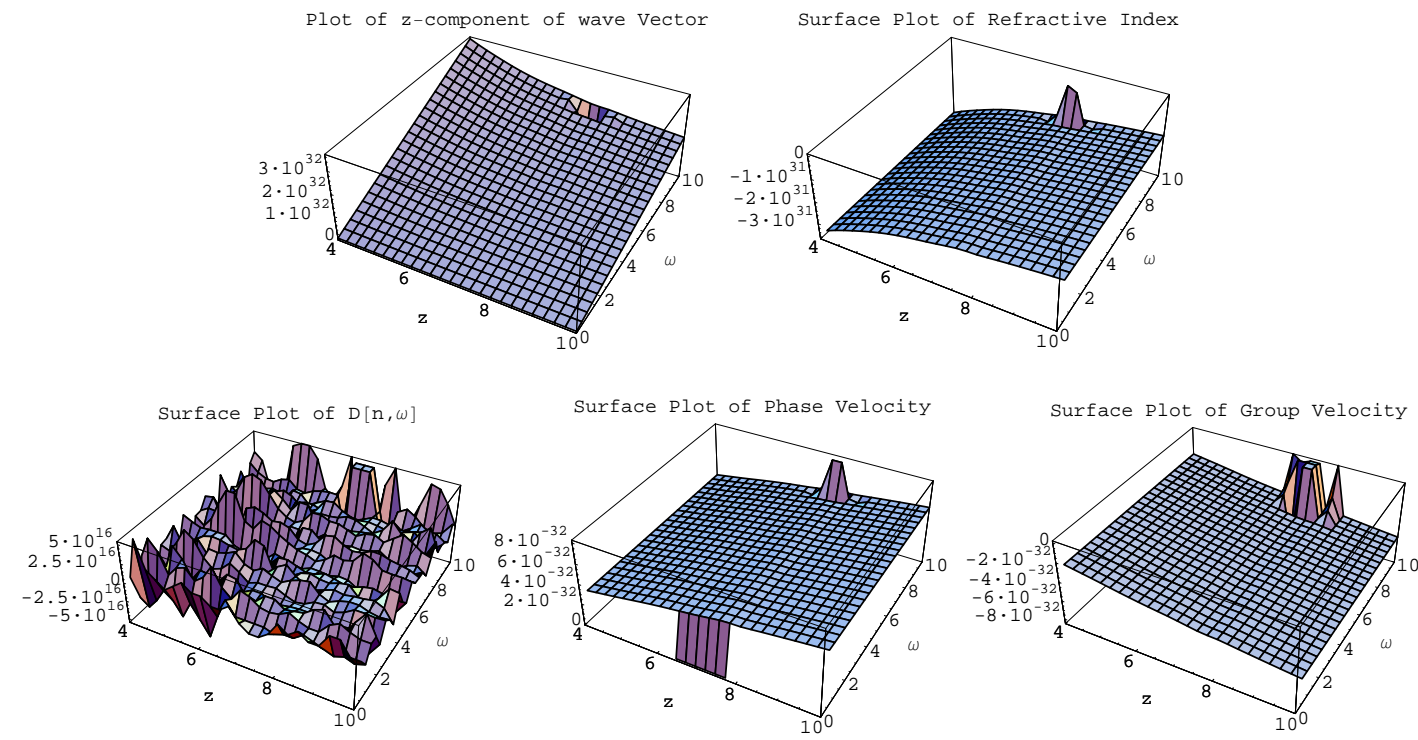

Figure 10: Waves exhibit normal as well as normal dispersion at random points.

The technique of Fourier analysis is applied to form dispersion relations for the rotating (non-magnetized and magnetized) plasma. Non-magnetized hot plasma indicates that waves in Figure $\mathbf{1}$ and $\mathbf{3}$ are directed towards black hole horizon and move away from the horizon in Figure $\mathbf{2}$. The dispersion is anomalous throughout the region in Figures $\mathbf{1}$ and $\mathbf{1 4}$ whereas it is normal in Figure 3.

In magnetized environment, waves are in the direction of event horizon in Figures 4-6 and 8-9 while they moves away from horizon in Figures $\mathbf{7}$ and 10. Normal as well as anomalous dispersion is observed in Figures 7-10 while it is anomalous in Figure 5 and normal in Figures 4 and $\mathbf{6}$.

In conventional refraction, $n$ is always greater than one, however, it is negative for Veselago medium. Phase velocity should be greater than group velocity in unusual medium and so in all the Figures refractive index is less than one. Index increases and decreases in small regions, dispersion is normal when $\frac{d n}{d \omega}$ is positive and anomalous otherwise. All the roots of dispersion relation in non-magnetize and magnetize plasmas (isothermal and hot) satisfy unusual properties of Veselago medium. Results reassert presence of such 
unusual medium in RN-dS magnetosphere.

It is followed by previous work [31] that inclusion of positive cosmological constant in non-rotating black hole may reveal more information about magnetosphere. Here we have inspected that addition of charge and cosmological constant is beneficial to attain normal dispersion at majority of points in the region, however, most of the waves move towards event horizon. It may be concluded that spacetime charge affects the electromagnetic field firmly that waves are directed towards black hole horizon.

On comparison with recent manuscript [33] on waves around RN horizon, it is observed that addition of cosmological constant to charged black hole i.e., RN-dS spacetime provide more interesting results in terms of energy extraction information around magnetosphere. Hot plasma is more general-

ized form of plasma and it is reducible to isothermal plasma when specific enthalpy is considered to be constant.

\section{Appendix}

GRMHD equations are governed from basic MHD equations in account with Maxwells electrodynamical equations. 3+1 GRMHD equations for RN-dS metric in a Veselago medium become [31].

$$
\frac{\partial \mathbf{B}}{\partial t}=-\nabla \times(\alpha \mathbf{V} \times \mathbf{B}),
$$




$$
\begin{aligned}
& \nabla \cdot \mathbf{B}=0, \\
& \frac{\partial(\rho+p)}{\partial t}+(\rho+p)\left[\gamma^{2} \mathbf{V} \cdot \frac{\partial \mathbf{V}}{\partial t}+\gamma^{2} V \cdot(\alpha \mathbf{V} \cdot \nabla) \mathbf{V}+\nabla \cdot(\alpha \mathbf{V})\right]=0, \\
& \left\{\left((\rho+p) \gamma^{2}+\frac{\mathbf{B}^{2}}{4 \pi}\right) \delta_{i j}+(\rho+p) \gamma^{4} V_{i} V_{j}-\frac{1}{4 \pi} B_{i} B_{j}\right\}\left(\frac{1}{\alpha} \frac{\partial}{\partial t}\right. \\
& +\mathbf{V} \cdot \nabla) V^{j}+\gamma^{2} V_{i}(\mathbf{V} \cdot \nabla)(\rho+p)-\left(\frac{\mathbf{B}^{2}}{4 \pi} \delta_{i j}-\frac{1}{4 \pi} B_{i} B_{j}\right) V_{, k}^{j} V^{k} \\
& =-(\rho+p) \gamma^{2} a_{i}-p_{, i}+\frac{1}{4 \pi}(\mathbf{V} \times \mathbf{B})_{i} \nabla \cdot(\mathbf{V} \times \mathbf{B})-\frac{1}{8 \pi \alpha^{2}}(\alpha \mathbf{B})_{, i}^{2} \\
& +\frac{1}{4 \pi \alpha}\left(\alpha B_{i}\right)_{, j} B^{j}-\frac{1}{4 \pi \alpha}[\mathbf{B} \times\{\mathbf{V} \times(\nabla \times(\alpha \mathbf{V} \times \mathbf{B}))\}]_{i}, \\
& -\frac{1}{\alpha} \frac{\partial p}{\partial t}+(\rho+p)\left[\left(\frac{1}{\alpha} \frac{\partial}{\partial t}+\mathbf{V} \cdot \nabla\right) \gamma^{2}+2 \gamma^{2}(\mathbf{V} \cdot \mathbf{a})+\gamma^{2}(\nabla \cdot \mathbf{V})\right] \\
& -\frac{1}{4 \pi \alpha}(\mathbf{V} \times \mathbf{B}) \cdot\left[\left(\mathbf{V} \times \frac{\partial \mathbf{B}}{\partial t}\right)+\left(\mathbf{B} \times \frac{\partial \mathbf{B}}{\partial t}\right)-(\nabla \times \alpha \mathbf{B})\right]=0 .
\end{aligned}
$$

Component form of linearly perturbed GRMHD equation takes following form

$$
\begin{aligned}
& \frac{1}{\alpha} \frac{\partial b_{x}}{\partial t}-u b_{x, z}=\left(u b_{x}-v b_{z}-v_{x}+\lambda v_{z}\right) \nabla \ln \alpha \\
& -\left(v_{x, z}-\lambda v_{z, z}-\lambda^{\prime} v_{z}+v^{\prime} b_{z}+v b_{z, z}-u^{\prime} b_{x}\right) \\
& \frac{1}{\alpha} \frac{\partial b_{z}}{\partial t}=0 \\
& b_{z, z}=0, \\
& \rho \frac{1}{\alpha} \frac{\partial \tilde{\rho}}{\partial t}+p \frac{1}{\alpha} \frac{\partial \tilde{p}}{\partial t}+(\rho+p) \gamma^{2} v\left(\frac{1}{\alpha} \frac{\partial v_{x}}{\partial t}+u v_{x, z}\right)+(\rho+p) \gamma^{2} u \frac{1}{\alpha} \frac{\partial v_{z}}{\partial t} \\
& +(\rho+p)\left(1+\gamma^{2} u^{2}\right) v_{z, z}=-\gamma^{2} u(\rho+p)\left[\left(1+2 \gamma^{2} v^{2}\right) v^{\prime}+2 \gamma^{2} u v u^{\prime}\right] v_{x} \\
& +(\rho+p)\left[\left(1-2 \gamma^{2} u^{2}\right)\left(1+\gamma^{2} u^{2}\right) \frac{u^{\prime}}{u}-2 \gamma^{4} u^{2} v v^{\prime}\right] v_{z}, \\
& \left\{(\rho+p) \gamma^{2}\left(1+\gamma^{2} v^{2}\right)+\frac{B^{2}}{4 \pi}\right\} \frac{1}{\alpha} \frac{\partial v_{x}}{\partial t}+\left\{(\rho+p) \gamma^{4} u v-\frac{\lambda B^{2}}{4 \pi}\right\} \\
& \times \frac{1}{\alpha} \frac{\partial v_{z}}{\partial t}+\left\{(\rho+p) \gamma^{2}\left(1+\gamma^{2} v^{2}\right)+\frac{B^{2}}{4 \pi}\right\} u v_{x, z}+\left\{(\rho+p) \gamma^{4} u v\right.
\end{aligned}
$$




$$
\begin{aligned}
& \left.-\frac{\lambda B^{2}}{4 \pi}\right\} u v_{z, z}-\frac{B^{2}}{4 \pi}\left(1+u^{2}\right) b_{x, z}-\frac{B^{2}}{4 \pi \alpha}\left\{\alpha^{\prime}\left(1+u^{2}\right)+\alpha u u^{\prime}\right\} b_{x} \\
& +\gamma^{2} u(\rho \tilde{\rho}+p \tilde{p})\left\{\left(1+\gamma^{2} v^{2}\right) v^{\prime}+\gamma^{2} u v u^{\prime}\right\}+\gamma^{2} u v\left(\rho^{\prime} \tilde{\rho}+\rho \tilde{\rho}^{\prime}\right. \\
& \left.+p^{\prime} \tilde{p}+p \tilde{p}^{\prime}\right)+\left[(\rho+p) \gamma^{4} u\left\{\left(1+4 \gamma^{2} v^{2}\right) u u^{\prime}+4 v v^{\prime}\left(1+\gamma^{2} v^{2}\right)\right\}\right. \\
& \left.+\frac{B^{2} u \alpha^{\prime}}{4 \pi \alpha}+\gamma^{2} u\left(1+2 \gamma^{2} v^{2}\right)\left(\rho^{\prime}+p^{\prime}\right)\right] v_{x}+\left[( \rho + p ) \gamma ^ { 2 } \left\{\left(1+2 \gamma^{2} u^{2}\right)\right.\right. \\
& \left.\left(1+2 \gamma^{2} v^{2}\right) v^{\prime}-\gamma^{2} v^{2} v^{\prime}+2 \gamma^{2}\left(1+2 \gamma^{2} u^{2}\right) u v u^{\prime}\right\}-\frac{B^{2} u}{4 \pi \alpha}(\lambda \alpha)^{\prime} \\
& \left.+\gamma^{2} v\left(1+2 \gamma^{2} u^{2}\right)\left(\rho^{\prime}+p^{\prime}\right)\right] v_{z}=0 \text {, } \\
& \left\{(\rho+p) \gamma^{2}\left(1+\gamma^{2} u^{2}\right)+\frac{\xi^{2} B^{2}}{4 \pi}\right\} \frac{1}{\alpha} \frac{\partial v_{z}}{\partial t}+\left\{(\rho+p) \gamma^{4} u v-\frac{\xi B^{2}}{4 \pi}\right\} \\
& \times \frac{1}{\alpha} \frac{\partial v_{x}}{\partial t}+\left\{(\rho+p) \gamma^{2}\left(1+\gamma^{2} u^{2}\right)+\frac{\xi^{2} B^{2}}{4 \pi}\right\} u v_{z, z}+\left\{(\rho+p) \gamma^{4} u v\right. \\
& \left.-\frac{\xi B^{2}}{4 \pi}\right\} u v_{x, z}+\frac{\xi B^{2}}{4 \pi}\left(1+u^{2}\right) b_{x, z}+\frac{B^{2}}{4 \pi \alpha}\left\{(\alpha \xi)^{\prime}-\alpha^{\prime} \xi+u \xi\left(u \alpha^{\prime}+u^{\prime} \alpha\right)\right\} b_{x} \\
& +(\rho \tilde{\rho}+p \tilde{p}) \gamma^{2}\left\{a_{z}+u u^{\prime}\left(1+\gamma^{2} u^{2}\right)+\gamma^{2} u^{2} v v^{\prime}\right\}+\left(1+\gamma^{2} u^{2}\right)\left(p^{\prime} \tilde{p}+p \tilde{p}^{\prime}\right) \\
& +\gamma^{2} u^{2}\left(\rho^{\prime} \tilde{\rho}+\rho \tilde{\rho}^{\prime}\right)+\left[( \rho + p ) \gamma ^ { 4 } \left\{u^{2} v^{\prime}\left(1+4 \gamma^{2} v^{2}\right)+2 v\left(a_{z}+u u^{\prime}(1+\right.\right.\right. \\
& \left.\left.\left.\left.2 \gamma^{2} u^{2}\right)\right)\right\}-\frac{\xi B^{2} u \alpha^{\prime}}{4 \pi \alpha}+2 \gamma^{4} u^{2} v\left(\rho^{\prime}+p^{\prime}\right)\right] v_{x}+\left[( \rho + p ) \gamma ^ { 2 } \left\{u^{\prime}\left(1+\gamma^{2} u^{2}\right)(1+\right.\right. \\
& \left.\left.4 \gamma^{2} u^{2}\right)+2 u \gamma^{2}\left(a_{z}+\left(1+2 \gamma^{2} u^{2}\right) v v^{\prime}\right)\right\}+\frac{\xi B^{2} u}{4 \pi \alpha}(\alpha \xi)^{\prime}+2 \gamma^{2} u(1 \\
& \left.\left.+\gamma^{2} u^{2}\right)\left(\rho^{\prime}+p^{\prime}\right)\right] v_{z}=0 \\
& \frac{1}{\alpha} \gamma^{2} \rho \frac{\partial \tilde{\rho}}{\partial t}+\frac{1}{\alpha} \gamma^{2} p \frac{\partial \tilde{p}}{\partial t}+\gamma^{2}\left(\rho^{\prime}+p^{\prime}\right) v_{z}+u \gamma^{2}\left(\rho \tilde{\rho}_{, z}+p \tilde{p}_{, z}+\rho^{\prime} \tilde{\rho}+p^{\prime} \tilde{p}\right) \\
& -\frac{1}{\alpha} p \frac{\partial \tilde{p}}{\partial t}+2 \gamma^{2} u(\rho \tilde{\rho}+p \tilde{p}) a_{z}+\gamma^{2} u^{\prime}(\rho \tilde{\rho}+p \tilde{p})+2(\rho+p) \gamma^{4}\left(u V^{\prime}\right. \\
& \left.+2 u v a_{z}+u^{\prime} v\right) v_{x}+2(\rho+p) \gamma^{2}\left(2 \gamma^{2} u u^{\prime}+a_{z} \gamma^{4}+2 \gamma^{2} u^{2} a_{z}\right) v_{z}+ \\
& 2(\rho+p) \gamma^{4} u v v_{x, z}+(\rho+p) \gamma^{2}\left(1+2 \gamma^{2} u^{2}\right) v_{z, z}-\frac{B^{2}}{4 \pi \alpha}\left[\left(v^{2}+u^{2}\right) \xi \frac{\partial b_{x}}{\partial t}\right. \\
& \left.+\left(v^{2}+u^{2}\right) \frac{\partial b_{z}}{\partial t}-\xi V(\xi v+u) \frac{\partial b_{x}}{\partial t}-u(\xi v+u) \frac{\partial b_{z}}{\partial t}\right]-\frac{B^{2}}{4 \pi \alpha} \\
& \times\left[\xi(\xi v+u) v_{x, t}+(\xi v+u) v_{z, t}-\left(\xi^{2}+1\right) v v_{x, t}-\left(\xi^{2}+1\right) u v_{z, t}\right] \\
& +\frac{B^{2}}{4 \pi}\left(\xi \xi^{\prime} v_{z}-\xi^{\prime} v_{x}-\xi^{\prime} v b_{z}+\xi^{\prime} u b_{x}-v b_{x, z}+u \xi b_{x, z}\right)=0 .
\end{aligned}
$$




\section{References}

[1] Camenzind, M.: Compact Objects in Astrophysics: White Dwarfs, Neutron Stars and Black Holes (Springer, 2007).

[2] Baumjohann, W. and Treumann, R.A.: Basic Space Plasma Physics (Imperial College Press, 1997).

[3] Raine, D. and Thomas, E.: Black Holes: An Introduction (Imperial College Press, 2005).

[4] Reiss, A.G. et al.: Astron. 116(1998)1009.

[5] Bahcall, N.A., Ostriker, J.P., Perlmutter, S. and Steinhardt, P.J.: Science 284(1999)1481.

[6] Perlmutter, S. et al.: Bull. Am. Astron. Soc. 29(1997)1351.

[7] Brill, D. R. and Hayward, S. A.: Class.Quant.Grav. 11(1994)359.

[8] Regge, T. and Wheeler, J.A.: Phy. Rev. 108(1957)1063.

[9] Zerilli, F.J.: Phys. Rev. D2(1970)2141; J. Math. Phys. 11(1970)2203; Phys. Rev. Lett. 24(1970)737.

[10] Gleiser, R.J., Nicasio, C.O., Price, R.H. and Pullin, J.: Class. Quantum Grav. 13(1972)L117.

[11] Arnowitt, R., Deser, S. and Misner, C.W.: Gravitation: An Introduction to Current Research (John Wiley, 1962).

[12] Petterson, J.A.: Phys. Rev. D10(1974)3166.

[13] Zhang, X.-H.: Phys. Rev. D39(1989)2933.

[14] Zhang, X.-H.: Phys. Rev. D40(1989)3858.

[15] Buzzi, V., Hines, K.C. and Treumann, R.A.: Phys. Rev. D51(1995)6663; ibid. 6677.

[16] Astefanesei, D., Mann, R. and Radu, E.: JHEP 029(2001)0401.

[17] Zhong, C. and Gao, S.:JETP Letters 94(2011)589. 
[18] Ali, M.H. and Rahman, M.A.: Int. J. Theor. Phys. 48(2009)1717.

[19] Sharif, M. and Sheikh, U.: Gen. Relativ. Gravit. 39(2007)1437; ibid. 2095; Int. J. Mod. Phys. A23(2008)1417; J. Korean Phys. Soc. 52(2008)152; ibid. 53(2008)2198.

[20] Sharif, M. and Sheikh, U.: Class. Quantum Grav. 24(2007)5495; Canadian J. Phys. 87(2009)879; J. Korean Phys. Soc. 55(2009)1677.

[21] Sharif, M. and Rafique, A.: Astrophys. Space Sci. 325(2010)227.

[22] Veselago, V.G.: Sov. Phys. Usp. 10(1968)509.

[23] Smith, D.R. and Kroll, N.: Phys. Rev. Lett. 85(2000)2933.

[24] Lakhtakia, A., McCall, M.W., Weiglhofer, W.S., Gerardin, J. and Wang, J.: Arch. Elektr. Ueber. 56(2002)407.

[25] Bliokh, K.Y. and Bliokh, Y.P.: Physics-Uspekhi. 47(2004)393.

[26] Mackay, T.G. and Lakhtakia, A.: Current Science 90(2006)641.

[27] Valanju, P.M., Walser, R.M. and Valanju, A.P.: Phys. Rev. Lett. 88(2002)187401.

[28] Ziolkowski, R.W. and Heyman, E.: Phys. Rev. E. 64(2001)056625.

[29] Ramakrishna, S.A.: Rep. Prog. Phys. 68 (2005)449.

[30] Sharif, M. and Mukhtar, N.: Astrophys. Space Sci. 331(2011)151; ibid 333(2011) 187

[31] Sharif, M. and Noureen, I.: Astrophys. Space Sci. 340(2012)185. ;Canadian J. Phys. 89(2011)991.

[32] Achenbach, J.D.: Wave Propagation in Elastic Solids (North-Holand Publishing Company, 1973).

[33] Ali, M.H. and Khan, M.A.: Astrophys. Space Sci. 348(2013)163. 